\title{
Dummy source digitization algorithm for reconstruction of flexible brachytherapy catheters with biplane images
}

\author{
Jenö Pálvölgyi, MSc, PhD \\ Petz County Teaching Hospital Department for Oncoradiology, Gyor, Hungary
}

\begin{abstract}
Purpose: The traditional brachytherapy catheter reconstruction with biplane images is based on digitizing radioopaque markers with a pointing device on a film or on a screen. An algorithm to automate digitization of radio-opaque marker coordinates on biplane images is presented.

Material and methods: To obtain the marker coordinates in a proper sequence, instead of usual pair of reconstruction images, series of images were taken with insertion of radio-opaque markers consecutively into the catheters. The images were pre-processed to suppress the shield of anatomic structures. The determination of the marker coordinates is based on the detection of characteristic high gradient variation in pre-processed image profiles. The method was tested with six endometrial insertions performed with Simon-Norman catheters using our version of Heyman packing.

Results: 28 catheters of six treatment fractions were digitized, typically 10 markers per catheter. To obtain the marker coordinates, adjustment of two threshold levels on the pre-processed images were needed. The coordinates of the radio-opaque markers on the biplane projection images were obtained without positive or negative artefact.

Conclusions: The dummy source coordinates on the biplane images were digitized in a proper sequence: from the catheters' tip towards the end of the catheters. After the three-dimensional reconstruction of the catheters from the digitized coordinates, the geometry file was imported by the brachytherapy planning system for dose calculation. The method has the advantage to eliminate manual digitization of the dummy sources.
\end{abstract}

Key words: automatic catheter digitization, brachytherapy, carcinoma of the endometrium.

\section{Purpose}

Multiple flexible brachytherapy catheters are applied by inserting the breast with endometrial treatments, using modified Heyman packing method with inflatable rectal applicator, and with surface insertions. Reconstruction of large number of catheters with biplane projection images is a time consuming process. More methods were published to speed up treatment planning with decreasing number of digitized points, and with elimination of the need of one-two-one coordinate correspondence requirement [1]. The use of automated applicator reconstruction has not become a common practice with the commercial brachytherapy planning systems. Only two methods were published in this field: one of them by Li et al. for reconstruction of Fletcher-Suit applicator [2], and the other by Rosenwald for localization of curved wires [3].

The modified Heyman packing according to Herbolsheimer et al., usually is performed with 6-11 pieces of Simon-Norman catheters. The heads of the catheters are uniformly distributed in the uterine cavity [4]. At our institution, endometrial insertions with smaller amount of
Simon-Norman catheters (Nucletron, an Elekta company, Elekta AB, Stockholm, Sweden) are performed. Another significant factor is the heads of each Simon-Norman catheters are positioned to the fundus uteri [5] as illustrated in Figure 1. Depending on the dimension of the uterus, the number of catheters is usually between 5 or 6 . The dose distributions are optimized with dwell times to fit the uterine contour [6]. The geometrical arrangement of the actual insertion approximates the ideal one illustrated in Figure 1. It differs more or less from that, therefore we prepare individual treatment plan for each treatment fraction. Even with reduced number of catheters, the number of points to digitize is over 50. An algorithm to automate the digitization of radio-opaque markers inserted into the Simon-Norman catheters is presented.

\section{Material and methods}

\section{Obtaining biplane reconstruction images}

At our institution, the reconstruction images are obtained with image capture from the video signal of a mo- 
bile C-arm image intensifier (Giraldoni, Italy). With the small power $\mathrm{C}$-arm, better image quality was experienced using PO images between 150 degrees and 142.5 degrees, than with usual lateral ones [7]. The fluoroscopy image size was $512 \times 512$ pixels with $0.32 \times 0.32 \mathrm{~mm}$ resolution, the pixel values were represented with 8 bits. The images were inverted, therefore the markers are appeared with high pixel values. The Simon-Norman catheters inserted into the uterus are close together and we experience overlap on the reconstruction images. The traditional biplane imaging was not suitable for the digitizing algorithm. To obtain the marker coordinates in a proper sequence (first catheter: first marker... $\mathrm{n}^{\text {th }}$ marker, second catheter: first marker... $\mathrm{n}^{\text {th }}$ marker, etc.), we have taken series of PA and PO images with markers inserted consecutively into one of the catheters. One of the images is illustrated in Figure 2A.

\section{Digitization of the marker coordinates on the reconstruction images}

Before digitizing the dummy sources, we suppress the pixel values of anatomical structures as follows. We obtain two copies of each reconstruction image: one of them is translated laterally by +1 pixel, while the other by -1 pixel, and the two images are subtracted. On the reconstruction image, the neighbouring pixel values is similar, therefore the anatomical structures on the differential image are suppressed (see Figure 2B). In contrary, the profiles taken across the markers show high gradient variation as illustrated in Figure 2C. The maxima and minima of the profile at the marker vary with shielding of bony structures, and with decreasing sensitivity of the image intensifier at the image circumference. On the differential images, a low and a high threshold levels are

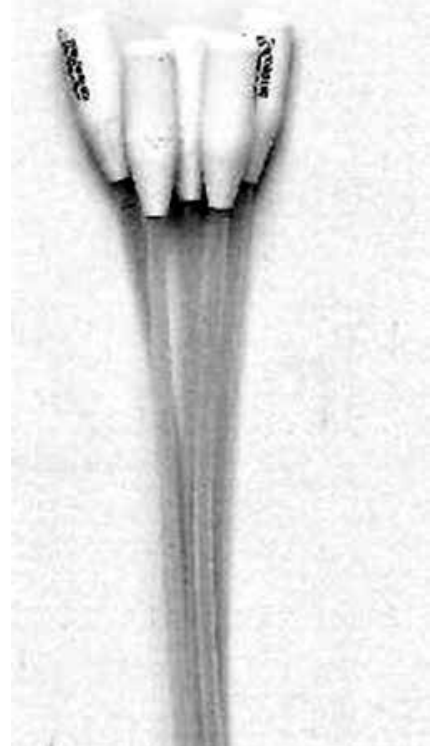

Fig. 1. Our version of Heyman packing: ideal arrangement of five Simon-Norman catheters with $6 \mathrm{~mm}$ diameter heads, each catheter is inserted to the fundus uteri

adjusted until each radio-opaque marker appears on the image without artefacts (see Figures $2 \mathrm{~B}$ and D) coordinates of the markers, obtained with an in-house written scanner program, which reads the differential image rows consecutively and searches for minima and maxima (' $M$ ' and ' $\mathrm{m}$ ' in Figure 2C) of the profiles between the sections determined by the two threshold levels. The location of a radio-opaque marker on an image row is the mean value of position of minima and maxima. Depending on the length of the radio-opaque marker,

A

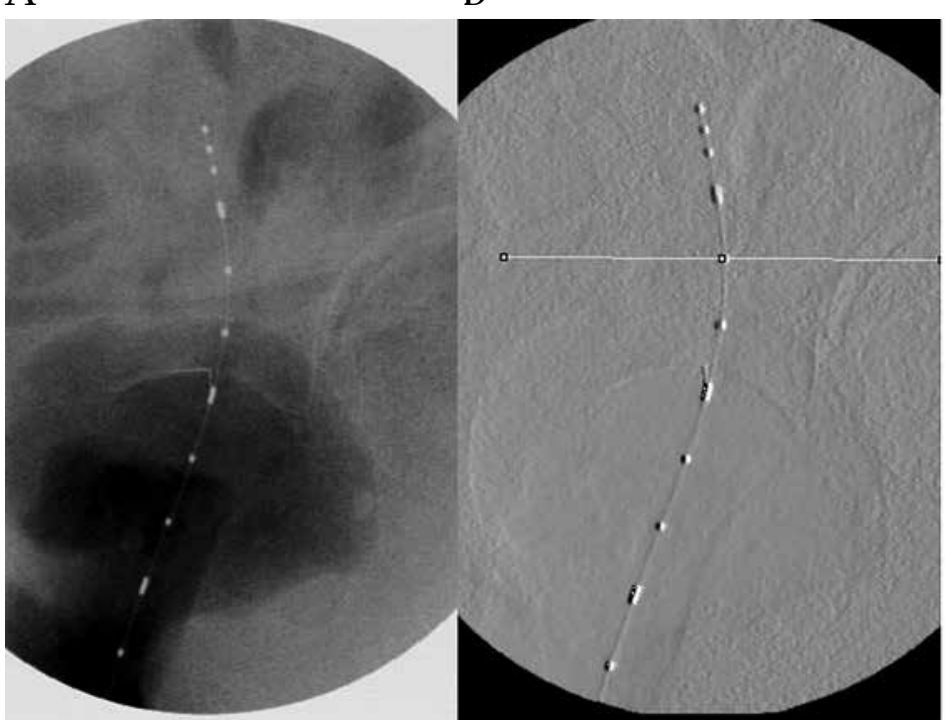

C

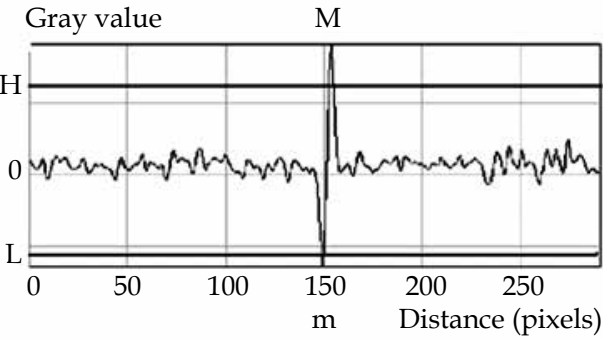

$\mathrm{D}$

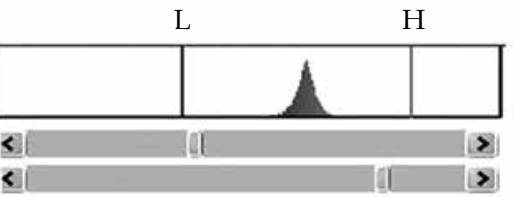

Fig. 2. Posterior-oblique reconstruction image of radio-opaque markers inserted into a Simon-Norman catheter (A), the difference image with a low and a high $(\mathrm{L}$ and $\mathrm{H})$ threshold levels $(\mathrm{B})$, the pixel values smaller than the low threshold level are represented with black, while pixel values larger than the high one are showed with white. Profile obtained across a marker along the section shown on the differential image (C) and histogram of the pixel values, and the threshold levels (D) 
the position is detected on 2-15 consecutive profiles. The coordinates of a radio-opaque marker are computed by averaging the numbers of the consecutive rows, where the marker is detected and by averaging the corresponding lateral position values. The image manipulations are performed with the ImageMagick software package (http://www.imagemagick.org), and the differential images with the actual threshold levels are visualized with the ImageJ program http:/ / www.rsbweb.nih.gov/ij).

\section{Determination of the optimal translation}

To determine the optimal value of translation of the reconstruction images, we performed the image processing with shifts by 1 to 5 pixels. The standard deviation of the pixel values in a region out of the markers $(N)$, the difference $(S)$ of the maxima $(M)$ and minima $(m)$ of the profiles taken across a selected marker, and the ratio $\mathrm{S} / \mathrm{N}$ on the differential images were computed and illustrated in Figure 3.

\section{The influence of the catheter inclination}

The Simon-Norman applicators follow the shape of the uterine cavity, therefore part of the catheters are not perpendicular to the image profiles. We simulated the catheter inclination with rotating the reconstruction image of a strait catheter through 15, 30, and 45 degrees. The number of marker detections in consecutive image profiles was counted and summarized in Table 1.

\section{The influence of the image quality}

The quality of the reconstruction images taken with the corpulent patient is often poor. We investigated the influence of the image noise on the digitizing process. We obtained noisy images from a good quality reconstruction image with adding noise with the ImageJ pro-

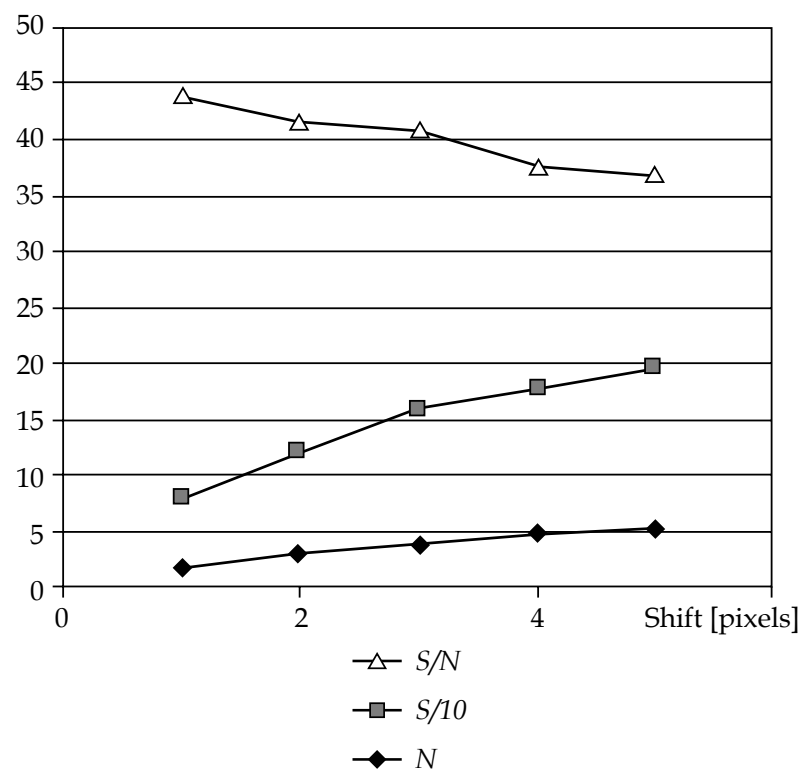

Fig. 3. The signal amplitude $(S)$ and the standard deviation of fluctuation $(N)$, and $S / N$ of the differential image versus the translation of the reconstruction image gram illustrated in Figure 4 . The $S / N$ values on the corresponding differential images (A...D) were: 34, 28, 12.8, and 10 , respectively.

\section{Testing the method with Simon-Norman applicators}

The marker digitizing method was tested in insertions with 3-6 pieces of Simon-Norman catheters of $6 \mathrm{~mm}$ diameter head, as illustrated in Figure 1. Since the original markers supplied with the Simon-Norman catheter set were not suitable for the method, we replaced them with radio-opaque markers $1 \mathrm{~cm}$ apart. We digitized 28 catheters of 6 insertions, and 10 markers per catheters. We computed the quantities $S, S / N$ and the difference of the high and low threshold levels $(H-L)$. These quantities were averaged for the PA and the PO images separately summarized in Table 2 .

\section{Results}

The quantities $S$ and $N$ of the differential images increased with the value of the translation, while the ratio $S / N$ decreased. The optimal value of translation was two pixels. Inclination of the catheter through 45 degrees decreased the number of marker detections in consecutive profiles by less than 3; the corresponding uncertainty was \pm 1 pixel. The lateral uncertainty of the marker positions of the non-inclined catheter was also \pm 1 pixel. The $S / N$ values of the images in Figure 4 were 28.7, 24.7, 12.8, and 10, respectively. The markers were recognized without artefact on the images A, B and C in Figure 4, while there were a missed marker and more artefacts on the very noisy image D. In insertions performed with the Simon-Norman catheters, the PA reconstruction images showed good or medium quality $(S / N>60$ and $S / N>30$, respectively), while the quality of the PO images was poorer. All markers were recognised without positive or negative artefact, the short markers in 2-4 consecutive profiles, while the long ones in 10-14 profiles. The difference between the high and low threshold levels $H$ - $L$ were 30 times the value of $N$ for good quality images, which decreased to 20 and 10 for medium, and poor ones.

Table 1. The number of marker detections in consecutive image profiles with rotated catheter

\begin{tabular}{ccccc}
$\begin{array}{c}\text { Marker } \\
\text { No. }\end{array}$ & $\begin{array}{c}0 \\
\text { degree }\end{array}$ & $\begin{array}{c}15 \\
\text { degrees }\end{array}$ & $\begin{array}{c}30 \\
\text { degrees }\end{array}$ & $\begin{array}{c}45 \\
\text { degrees }\end{array}$ \\
\hline 1 & 6 & 5 & 4 & 4 \\
\hline 2 & 6 & 5 & 4 & 3 \\
\hline 3 & 6 & 5 & 4 & 3 \\
\hline 4 & 11 & 11 & 10 & 8 \\
\hline 5 & 5 & 3 & 3 & 3 \\
\hline 6 & 12 & 10 & 10 & 9 \\
\hline 7 & 5 & 5 & 5 & 3 \\
\hline 8 & 6 & 4 & 3 & 4
\end{tabular}




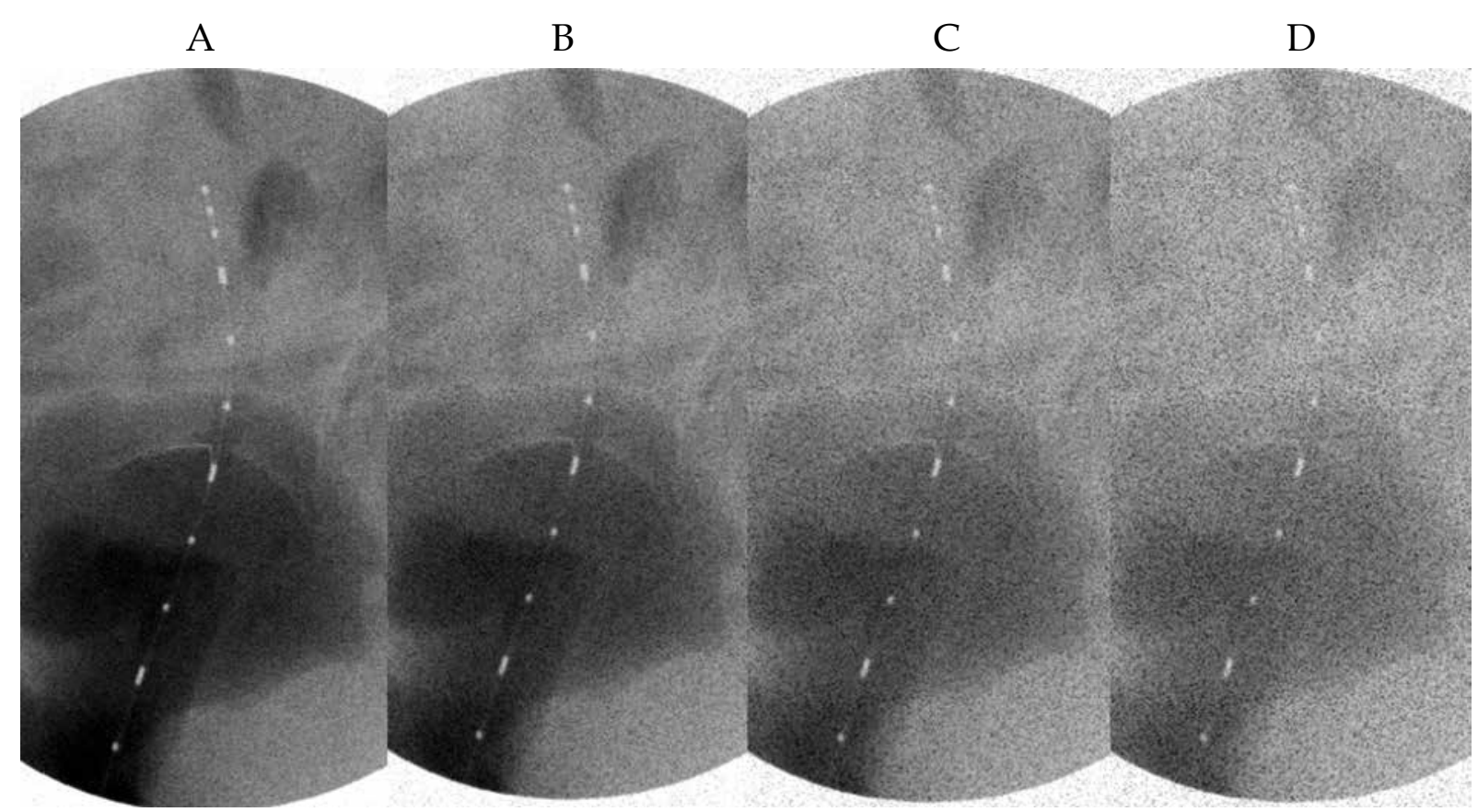

Fig. 4. Reconstruction images with added noise

\section{Discussion}

Weitman et al. described the use of the modified Heyman packing with MR images. They inserted 11 pieces of Simon-Norman catheters that were uniformly distributed in the uterus. Since the catheters identification was not possible with sectional images, the catheter reconstruction was performed with biplane X-ray images. The reconstructed coordinates were combined with the contours obtained with the MR [9]. At our institution, the endometrial treatments are performed with 5 or 6 piec- es of catheters positioned to the fundus uteri, the most probable location of the endometrial cancer. In our previous study, we showed that with this method higher dose can be delivered to the fundus uteri than with the Y shaped applicator [5]. With dwell time optimization, acceptable dose coverage can be achieved for the medium size uterus [6]. In lack of access to sectional imager, we apply a C-arm as a low cost alternative of the integrated brachytherapy unit. We capture the fluoroscopy images and convert to DICOM format, and store to a pictorial archive. Since the brachytherapy planning systems (BPS)

Table 2. The quantities $N, S / N$ and the difference of the high, and the low threshold levels $H$ - $L$ averaged for the $\mathrm{PA}$ and $\mathrm{PO}$ images in six Heyman packing fractions

\begin{tabular}{|c|c|c|c|c|c|c|c|c|}
\hline $\begin{array}{l}\text { Patient\#. } \\
\text { Fraction\# }\end{array}$ & \multicolumn{2}{|c|}{$\begin{array}{l}\text { Number of } \\
\text { catheters }\end{array}$} & $\begin{array}{c}\text { Number of } \\
\text { average }\end{array}$ & St.dev & $S / N$ average & St.dev & $H$ - $L$ average & St.dev \\
\hline \multirow{2}{*}{1.1} & 4 & PA & 3.2 & 0.1 & 35.9 & 1.8 & 56.5 & 2.1 \\
\hline & & $\mathrm{PO}$ & 4.5 & 0.2 & 25.2 & 2.3 & 50.0 & 3.9 \\
\hline \multirow{2}{*}{1.2} & 5 & PA & 3.1 & 0.0 & 30.7 & 3.2 & 41.2 & 6.3 \\
\hline & & $\mathrm{PO}$ & 4.1 & 0.2 & 21.1 & 4.5 & 42.7 & 6.7 \\
\hline \multirow{2}{*}{2.1} & 6 & $\mathrm{PA}$ & 1.8 & 0.1 & 62.4 & 6.8 & 63.7 & 7.9 \\
\hline & & $\mathrm{PO}$ & 2.6 & 0.1 & 62.4 & 6.8 & 59.5 & 4.5 \\
\hline \multirow{2}{*}{3.1} & 5 & $\mathrm{PA}$ & 2.7 & 0,3 & 46.1 & 5.9 & 56.6 & 3.2 \\
\hline & & $\mathrm{PO}$ & 3.0 & 0,1 & 37.1 & 6.3 & 66.8 & 2.4 \\
\hline \multirow{2}{*}{4.1} & 5 & PA & 2.2 & 0.1 & 61.8 & 3.8 & 51.2 & 3.9 \\
\hline & & $\mathrm{PO}$ & 1.8 & 0.1 & 69.9 & 4.9 & 57.0 & 3.6 \\
\hline \multirow{2}{*}{5.1} & 3 & PA & 1.9 & 0.4 & 65.4 & 13,3 & 61.3 & 4,7 \\
\hline & & $\mathrm{PO}$ & 2.0 & 3.5 & 48.6 & 5.2 & 49.0 & 7.0 \\
\hline
\end{tabular}


do not allows to import the dummy sources coordinates, therefore we reconstructed the three-dimensional coordinates of the catheters. However, analytical solution can be applied in this case and we used our multi-parametric fit method [8]. After reconstruction of each catheter, we generated a geometry file of 3D coordinates, which was imported by the Plato BPS v13.7 (Nucletron, an Elekta company, Elekta AB, Stockholm, Sweden) for dose calculation.

\section{Conclusions}

The coordinates for the three-dimensional reconstruction of the dummy sources should be obtained with a proper sequence on the reconstruction images. Since the Simon Norman catheters are close together and often overlapped, this requirement was ensured with taking a series of biplane images with markers inserted consecutively into the catheters. The images were pre-processed and scanned with profiles close perpendicular to the catheters. The dummy source coordinates were obtained from the catheters' tip towards the end. Small intervention of the user to adjust the threshold levels on the pre-processed images was required. However, for the method a series of images, which number is two times the number of catheters is needed, has the advantage of elimination of manual digitization of large number of marker points and ensures accurate reconstruction of Simon Norman catheters.

\section{Acknowledgements}

The Heyman packing insertions for the study were performed by Kofi Agyemang-Prempeh M.D.

\section{Disclosure}

Author reports no conflict of interest.

\section{References}

1. Li S, Chen GTY, Pelizzari CA et al. A new source localization algorithm with no requirement of one-to-one Source correspondence between biplane radiographs. Med Phys 1996; 23: 921-927.

2. Li S, Pellizzari CA, Reft C et al. Computer-aided reconstruction of Fletcher-Suit Source positions. Med Phys 1994; 21: 1123-1130.

3. Rosenwald JC. Automatic localization of curved wires used in brachytherapy. Comput Prog Biomed 1975; 4: 103-112.

4. Herbolsheimer M, Sauer O. HDR brachytherapy for endometrial cancer. International Nucletron Radiotherapy Journal 1996; Special Report No.8: 34-45.

5. Pálvölgyi J, Agyemang-Prempeh K. Modified Heyman packing performed with small number of Simon-Norman catheters. J Contemp Brachytherapy 2010; 2: 33-36.

6. Pálvölgyi J. Dose Distribution of Modified Heyman Packing. Physica Medica 2006; 22: 127-130.

7. Pálvölgyi J. To what extent can digital images obtained with a non-isocentric C-arm be used for brachytherapy treatment planning in gynaecology. Radiother Oncol 2006; 67: 107-112.

8. Palvolgyi J. Multiparametric fit method in reconstruction of Fletcher-Suit-Delclos applicator. Med Phys 2006; 33: 69-75.
9. Weitmann HD, Pötter R, Waldhaus C et al. Treatment of endometrial carcinoma with $3 \mathrm{D}$ image-based high dose rate brachytherapy using modified Heyman packing: clinical experience and dose volume histogram analysis. Int J Radiat Oncol Biol Phys 2005; 62: 468-478. 